\title{
TAMeless traitors: macrophages in cancer progression and metastasis
}

\author{
Shweta Aras $^{1}$ and M Raza Zaidi ${ }^{\star}, 1$ \\ ${ }^{1}$ Fels Institute for Cancer Research and Molecular Biology, and Department of Medical Genetics and Molecular Biochemistry, \\ Lewis Katz School of Medicine at Temple University, Philadelphia, PA 19140, USA
}

\begin{abstract}
Macrophages are conventionally classified into M1 and M2 subtypes according to their differentiation status and functional role in the immune system. However, accumulating evidence suggests that this binary classification system is insufficient to account for the remarkable plasticity of macrophages that gives rise to an immense diversity of subtypes. This diverse spectrum of macrophage subtypes play critical roles in various homeostatic and immune functions, but remain far from being fully characterised. In addition to their roles in normal physiological conditions, macrophages also play crucial roles in disease conditions such as cancer. In this review, we discuss the roles tumour-associated macrophages (TAMs) play in regulating different steps of tumour progression and metastasis, and the opportunities to target them in the quest for cancer prevention and treatment.
\end{abstract}

Macrophages constitute a prominent set of immune cells that are phagocytic in nature and are present in almost all tissues. In general, they are differentiated cells of mononuclear origin and display specific phenotypic characteristics. In mice, macrophages show surface expression of markers such as CD11b, F4/80 and colony-stimulating factor-1 receptor (CSF-1R) and do not express Gr1; whereas, in humans, macrophages show expression of CD68, CD163, CD16, CD312 and CD115 (Qian and Pollard, 2010).

Macrophages are an incredibly diverse set of cells, constantly altering their functional state in response to environmental stimuli. They undergo the 'polarisation' process wherein they express different surface markers and functional programs in response to microenvironmental stimuli such as the cytokines and other signalling mediators. According to the binary polarisation concept, there exist two polarisation states of macrophages: Classically activated macrophages (M1) produce pro-inflammatory cytokines and reactive oxygen/nitrogen species, which are crucial for host defence and tumour cell killing and, therefore, are considered as 'good' macrophages. The alternatively activated macrophages (M2) produce anti-inflammatory cytokines and are involved in the resolution of inflammation. These are considered 'bad' macrophages because they not only suppress the destructive immunity against parasites and tumour cells, but also promote angiogenesis and matrix remodelling, which make the tumour microenvironment conducive to tumour progression and metastasis (Huang and Feng, 2013).

Macrophages not only perform vital functions in normal physiological conditions such as development, wound healing, infection and maintenance of tissue homeostasis, but are also involved in a variety of disease conditions such as autoimmune disorders, atherosclerosis and tumourigenesis (Wynn et al, 2013). Tumourigenesis is a highly complex, multi-step process and many findings provide strong evidence for the role of specific subsets of macrophages in tumourigenesis. These macrophages are commonly referred to as 'tumour-associated macrophages (TAMs)'. Tumour-associated macrophages were thought to closely resemble the M2 phenotype; however, findings discussed in this review suggest that this binary polarisation model is going obsolete, and there exists a whole spectrum of TAM phenotypes that are yet to be discovered and fully characterised. Tumour-associated macrophages also contribute to many steps of tumourigenesis, such as transformation, tumour cell proliferation, angiogenesis, invasion and metastasis. In this review, we summarise various physiological and functional aspects of TAMs, as well as their roles in regulating various steps of tumour initiation, progression and metastasis. This review will help shed light on the potential of TAMs as prognostic biomarkers for various cancers, as well as ways to target them for therapeutic interventions.

\section{THE TWO FACES OF MACROPHAGES}

The role of macrophages in tumourigenesis has been controversial. Macrophages have conventionally been considered to be antitumourigenic in nature, play tumour-suppressive roles, and 
illustrate a significant link between high density and better prognosis, particularly in the case of colorectal cancer. For instance, Ong et al showed that macrophages in a colorectal cancer model are pro-inflammatory, and inhibited the growth of tumour cells by secreting chemokines to attract $\mathrm{T}$ cells, thereby priming an anti-tumour type-1 inflammatory response (Ong et al, 2012). It was also reported that when stimulated with TLR ligands, anti-CD40 or interferon- $\gamma$ (IFN- $\gamma)$, TAMs show anti-tumoural functions, provided CD47 expression on cancer cells does not inhibit tumour cell phagocytosis (Jaiswal et al, 2009). Moreover, pro-inflammatory macrophages were also shown to have tumour suppressive effects via production of reactive oxygen species and reactive nitrogen intermediates (Vicetti Miguel et al, 2010). However, many subsequent studies challenged this notion and indicated that macrophages also display pro-tumourigenic properties. In a mouse model of breast cancer, Lin et al showed that a homozygous null mutation of a gene encoding the macrophage growth factor, colony stimulating factor-1 (CSF-1), not only reduced macrophage infiltration but also completely abolished tumour progression and metastasis. On the contrary, overexpression of this CSF-1 protein increased the rate of tumour progression and metastasis (Lin et al, 2001). Moreover, inhibition of the CSF-1 receptor signalling pathway by virtue of a small molecule inhibitor abrogated infiltration of TAMs and enhanced recruitment of $\mathrm{CD} 8+\mathrm{T}$ cells, thereby reducing cervical and mammary tumour growth (Strachan et al, 2013). According to Shree et al, cathepsinexpressing macrophages protected against chemotherapy-induced tumour cell death in breast cancer, and cathepsin inhibition significantly reversed this phenomenon (Shree et al, 2011). Recently, Gordon et al, showed that both mouse and human TAMs express programmed cell death protein 1 (PD-1), thereby negatively regulating their phagocytic activity against tumour cells. Blockade of this PD-1/PD-L1 axis restores phagocytic activity by these TAMs, reduces tumour growth and lengthens survival of mice, strongly suggesting a pro-tumourigenic potential of these TAMs (Gordon et al, 2017).

Macrophages are multifaceted and highly plastic in their characteristics. The classically activated M1 macrophages are stimulated by microbial substrates such as lipopolysaccharide, toll-like receptor ligands and cytokines such as IFN- $\gamma$, and are involved in Th1 type of responses. Once activated, M1 macrophages are characterised by secretion of pro-inflammatory cytokines such as interleukins IL6, IL12, IL23 and tumour necrosis factor- $\alpha$, and have a strong microbicidal and tumouricidal functions. Phenotypically, they express high levels of major histocompatibility complex class II (MHC-II), CD68, and CD80 and CD86 costimulatory molecules.
The alternatively activated M2 macrophages are stimulated by IL4 and IL13, secrete IL10, transforming growth factor- $\beta$ (TGF- $\beta$ ), and chemokines, and are involved in tissue remodelling and tumour progression (Fan et al, 2016). Phenotypically, M2 macrophages express low levels of MHC-II and feature expression of CD163 (Barros et al, 2013), CD200R membrane glycoprotein (Jaguin et al, 2013) as well as high levels of MGL1 and MGL2, which are members of the macrophage galactose type C-lectin family (Raes et al, 2005). A genetic profile for M2 macrophages showed upregulation of the genes arginase 1 (Arg1), MMR (Mrc1), resistin-like molecule $\alpha$ (FIZZ1), and chitinase-like protein $Y m 1$ (Raes et al, 2002). Tumour-associated macrophages in the tumour microenvironment exhibit M2-like polarisation state of macrophages with pro-tumourigenic functions because they express a series of markers, such as CD163, the Fc fragment of IgG, C-type lectin domains, and heat shock proteins, some of which are commonly expressed in M2-macrophages. Moreover, acquisition of an M2-like phenotype is also caused by secretion of tumourderived cytokines such as IL4, IL10, and IL13 (Sica et al, 2002; Sakai et al, 2008) (Figure 1).

\section{THE CLASSIFICATION CONUNDRUM}

A large body of research clearly suggests that the historical binary classification of macrophages is grossly oversimplified, and represents two extremes of their activation states. In view of some recent findings about macrophage activation, the classical M1/M2 polarisation model seems to be obsolete as it fails to fully account for the complexity of the macrophage activation process. Xue et al recently showed that by virtue of highly specific and standardised stimulation of human macrophages, the current M1/M2 paradigm can be expanded into a 'spectrum model' (Xue et al, 2014). This model suggests that due to the presence of a network of transcriptional regulators, there exists a spectrum of differentiated macrophages, many of which are yet to be fully discovered.

A recently published report identified a few other categories of macrophages with molecular phenotypes that do not fit the conventional M1 or M2 types, but have been involved as main players in various human pathologies. For example, the antigen CD169 (Siglec-1) is highly expressed on and is reported as a marker of one macrophage subpopulation found in bone marrow, lymph node, liver, and spleen. Although the information about the signalling pathway involved in the activation of CD169+ macrophages is imprecise, CD169+ macrophages are mainly involved in erythropoiesis and immune regulation (Chow et al, 2013). Another non-M1/M2 subtype of macrophages expresses

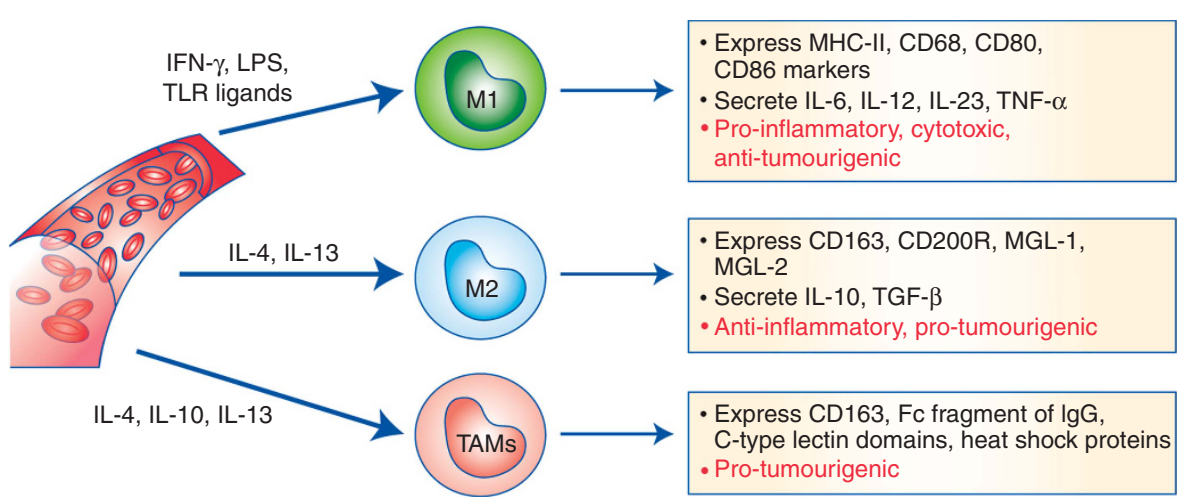

Figure 1. Macrophage differentiation and their role in tumourigenesis. Classically activated (M1) macrophages are activated by IFN- $\gamma$, LPS or TLR ligands, secrete pro-inflammatory cytokines and play tumouricidal roles. Alternatively activated (M2) macrophages are activated by IL-4 and IL-13, secrete anti-inflammatory cytokines IL-10 and TGF- $\beta$ and play tumourigenic roles. Tumour-associated macrophages (TAMs) display M2-like phenotype and exhibit pro-tumourigenic features. 
T-cell receptor (TCR). T-cell receptor is required for antigen recognition, and several reports have suggested the presence of murine and human TCR + macrophages, especially TCR $\alpha \beta^{+}$and $\mathrm{TCR} \gamma \delta^{+}$macrophages, in inflammatory and infectious diseases (Chavez-Galan et al, 2015). Georgoudaki et al recently identified a novel subtype of TAMs with an M2-like immunosuppressive gene profile expressing a novel receptor 'macrophage receptor with collagenous structure' or 'MARCO' in mouse tumour models of mammary carcinoma, colon cancer and B16 melanoma (Georgoudaki et al, 2016).

Another example of non-M1/M2-type macrophages is IFN- $\gamma$ secreting macrophages. Interferon- $\gamma$ forms an important constituent of the innate immune defence system and secretion of IFN- $\gamma$ by human NK cells and T cells in response to interleukins has been long established. However, IFN- $\gamma$ secretion by macrophages is still controversial owing to the doubts about contamination of macrophages by NK or T cells. However, a growing body of evidence suggests that macrophages are capable of secreting IFN- $\gamma$ both in vitro and in vivo. For instance, Darwich et al showed that at single cell level, human macrophages secrete IFN- $\gamma$ after induction with interleukins IL-12 and IL-18 (Darwich et al, 2009). Robinson et al showed a similar phenomenon of induction of IFN- $\gamma$ secretion post Mycobacterium tuberculosis infection of human macrophages (Robinson et al, 2010). We have also identified macrophages secreting IFN- $\gamma$, which promoted melanoma growth in an allograft mouse model (Zaidi et al, 2011) (Figure 2).

Given the importance of macrophages in homeostatic and pathological conditions, a thorough investigation of the multiple factors in normal and diseased microenvironments is absolutely warranted to dissect the mechanisms of macrophage activation, plasticity, and polarisation.

\section{TAMS IN TUMOUR MICROENVIRONMENT}

Tumour-associated macrophages originate from the circulating peripheral blood monocytes, which are derived from the bone marrow. These monocytes are recruited to the tumour tissues and then differentiate locally in response to a variety of cytokines, chemokines, and growth factors produced by the stromal and tumour cells in the tumour microenvironment. For instance, the chemokine CCL2 and macrophage colony-stimulating factor were shown to recruit inflammatory monocytes to the tumour site, and then differentiate into TAMs in response to IL-4, IL-10, IL-13 and other cytokines in the tumour microenvironment and promote tumour metastasis (Qian et al, 2011). Another report suggested that hypoxia-inducible chemotactic factors such as the CXCR4 ligand CXCL12 and Angiopoietin-2 (Ang-2) promote recruitment of Tie2-expressing monocytes in hypoxic areas of tumours and differentiate them into Tie2-expressing macrophages (Murdoch et al, 2007). Some other microenvironmental factors such as CSF-1, CCL2, IL6, vascular endothelial growth factor (VEGF-A) and platelet-derived growth factor (PDGF) have also been involved in infiltration of monocytes to the tumour sites (Balkwill, 2004; Joyce and Pollard, 2009).

It is now well-established that the majority of malignant tumours contain macrophages as a major component of their tumour microenvironment. Upon stimulation, these TAMs secrete a wide variety of cytokines, growth factors, inflammatory substrates and proteolytic enzymes that play major roles in cancer progression (Figure 3). Moreover, clinicopathological studies have shown that there also exists a strong correlation between increased macrophage density and poor prognosis in lung, hepatocellular carcinoma, renal cell carcinoma (Komohara et al, 2011) and breast cancer (Campbell et al, 2011; Mahmoud et al, 2012; Medrek et al, 2012). Moreover, according to a recent report by Wang et al, macrophages were also shown to play a critical role in melanoma resistance to BRAF inhibitors (Wang et al, 2015). Therefore, TAM infiltration can potentially be used as a prognostic marker of clinical outcomes for many cancers and can be potentially targeted for cancer prevention or treatment.

The leading or invasive edge of the primary tumour is a crucial site in the tumour microenvironment where immune cells and stromal cells are recruited and play immunosuppressive roles. TAMs are located at the perivascular areas or at the invasive edge of the tumours and are recruited there by tumour-derived chemoattractants. Upon arrival, TAMs supply pro-migratory factors such as epidermal growth factor (EGF), promote proteolytic remodelling of the extracellular matrix, accelerate tumour motility and induce migration and invasion of tumour cells (Quail and Joyce, 2013). According to a report by Wyckoff et al (2007), multiphoton microscopy of mouse mammary tumours showed

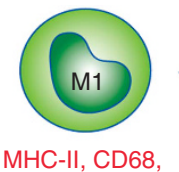
CD80 and CD86

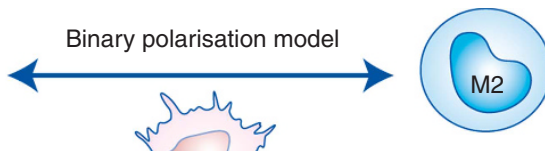

CD163, CD200R MGL-1, MGL-2

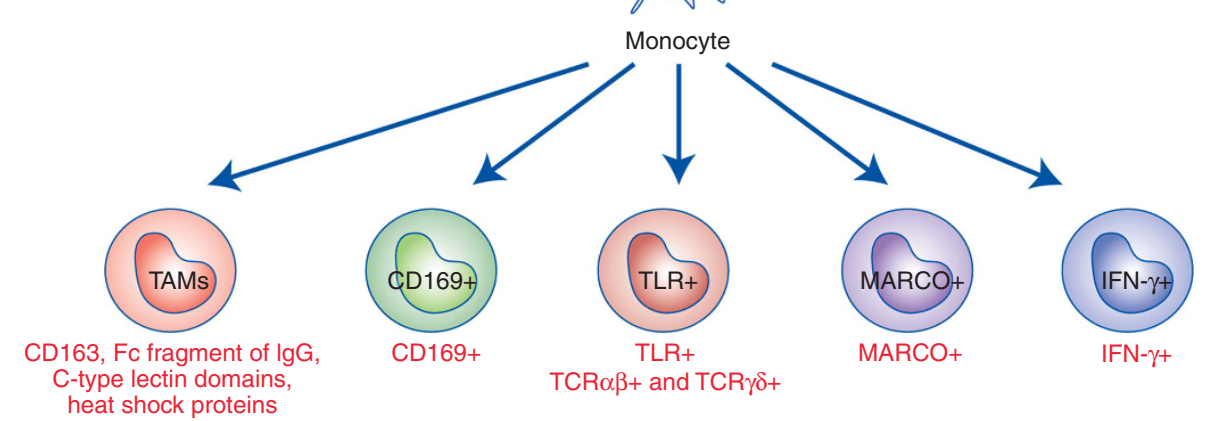

Spectral polarisation model

Figure 2. The 'binary' vs 'spectrum' model of macrophage polarisation. Recent evidence strongly suggests that the conventional model of binary polarisation of macrophages into M1 and M2 subtypes is oversimplified and the molecular profile of several newly discovered subtypes of macrophages do not fit either phenotype. The spectrum model of macrophage polarisation suggests that there exist various subtypes of differentiated macrophages by virtue of an intricate network of transcriptional regulators, which participate in many homeostatic as well as pathological functions. 


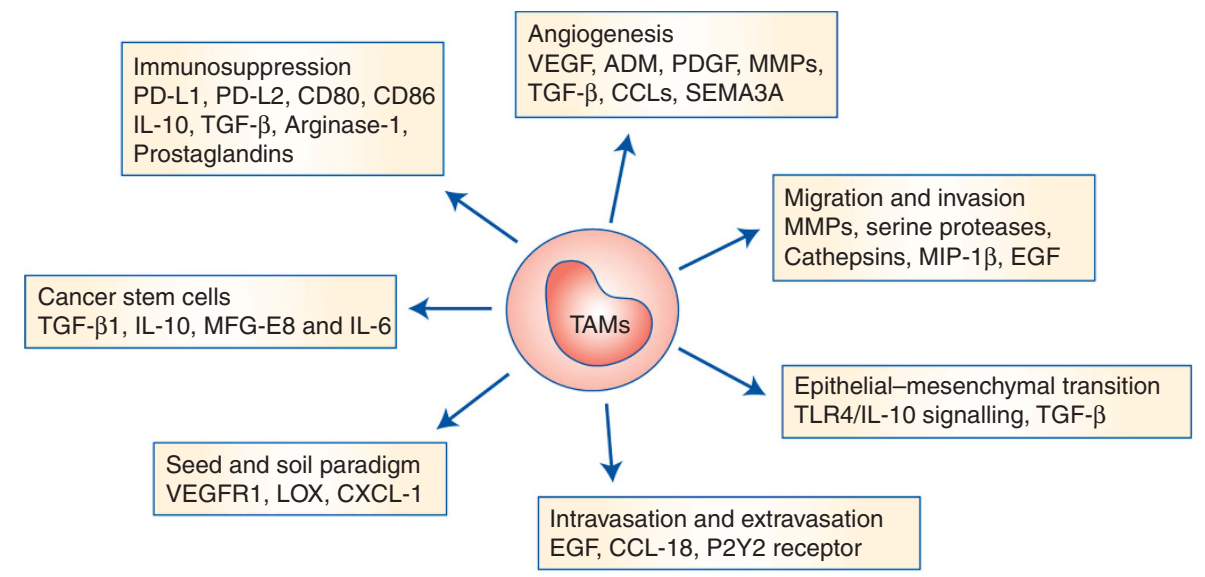

Figure 3. Role of TAMs in tumourigenesis. Different roles of TAMs in promoting tumour invasion and metastasis, along with the specific markers, are depicted. Tumour microenvironmental cues educate the macrophages to adopt a specific phenotype and perform distinct roles contributing towards tumourigenesis.

large number of macrophages at the margins of the tumours. Apart from the perivascular region of the tumour where they promote tumour cell invasion, TAMs are also reported to get recruited in the hypoxic regions of the tumour (Wyckoff et al, 2004).

The tumour microenvironment is a complex ecology of heterogeneous cell populations, which have a robust influence on tumourigenesis. For example, TAM-adipocyte interactions have been shown to drive cancer initiation and progression in case of obesity and overweight conditions. Adipocyte hypertrophy, inflammation and apoptosis results in macrophage recruitment, which phagocytose dead/dying adipocytes and develop inflammatory foci called crown-like structures (CLS). Many reports have shown that these adipocytes and apoptotic CLSs promote tumour progression in breast (Morris et al, 2011) and ovarian cancers (Nieman et al, 2011). Apart from TAMs, cancer-associated fibroblasts (CAFs) constitute another main component of infiltrating stromal cells that are reported to be involved in tumour progression (Komohara and Takeya, 2017). Recently, Hashimoto et al (2016) showed that cell-cell interaction between TAMs and CAFs promoted recruitment and activation of each other and contributed to neuroblastoma progression. Similarly, Miyake et al (2016) showed that high CXCL1 levels in urothelial cancer of the bladder cells resulted in enhanced recruitment of TAMs/CAFs, higher metastatic potential, and poor prognosis. In another report, CAFs were shown to promote an immunosuppressive microenvironment through the induction and accumulation of pro-tumoural macrophages, suggesting a strong crosstalk between microenvironmental stromal cells (Takahashi et al, 2017).

\section{TAMS IN ANGIOGENESIS}

In many cancers, benign-to-malignant transition is associated with a significant increase in vascularisation, a process known as angiogenesis, which provides cancer cells nutrients and oxygen to allow them to multiply, invade and metastasise. This process of forming new vasculature is highly complex and TAMs are one of the major contributors in this process (Lin and Pollard, 2007). According to a recent report, quantitative analysis and assessment of the spatial associations between TAMs and tumour neovasculature demonstrated the great significance and close association of TAMs and tumour angiogenesis during cervical cancer development and progression (Jiang et al, 2016). Neovascularisation is induced when TAMs secrete pro-angiogenic factors, such as VEGF, adrenomedullin (ADM), PDGF, TGF- $\beta$ and matrix metalloproteinases (MMPs). For instance, VEGF-A was reported to contribute to neoangiogenesis and macrophage recruitment at the tumour site in a mouse model of skin carcinogenesis (Linde et al, 2012). Moreover, TAMs were shown to sense hypoxia in avascular areas within tumours and release VEGF-A, a very potent pro-angiogenic factor (Laoui et al, 2014). Another report on Merkel cell carcinoma, a highly malignant neuroendocrine tumour of the skin, shows that TAMs express high levels of VEGF-C, which promotes lymphovascularisation (Werchau et al, 2012; Matsumoto-Okazaki et al, 2015). These reports strongly demonstrate the role of VEGF-producing TAMs in angiogenesis and tumour progression. Chen and colleagues found that infiltrating TAMs produced ADM when co-cultured with melanoma cells. There was also a significant improvement in endothelial cell proliferation and tube formation with $\mathrm{ADM}$ and this effect was abrogated upon administration of neutralising ADM antibody in vitro, suggesting the pro-angiogenic action of TAM-derived ADM (Chen et al, 2011b).

Apart from VEGFs and ADM, MMPs were also shown to be expressed by TAMs and involved in angiogenesis. According to a recent report, MMP9 secretion by TAMs recruited into the tumour site, in response to osteopontin signalling in melanoma, induced angiogenesis and tumour growth. These reports suggest that MMP9 aids tumour progression by remodelling the extracellular matrix and by promoting neoangiogenesis (Kale et al, 2015). Macrophage differentiation and chemotaxis is regulated by growth factors such as CSF1. Studies involving TAM depletion in breast tumours using Csf1-null mutation displayed substantial reduction in angiogenic potential and tumour burden, suggesting that these macrophages are required for angiogenesis. Additionally, this TAM depletion was reversed upon rescuing CSF1 in breast epithelium. Furthermore, overexpression of CSF1 in wild-type mice led to premature accumulation of macrophages in lesions and a dramatic increase in angiogenesis. It has also been demonstrated that most TAM depletion strategies using liposome-encapsulated clodronate inhibits angiogenesis in tumour models (Gazzaniga et al, 2007; Halin et al, 2009).

Hypoxia is a major contributor in angiogenesis. Dual staining of hypoxia and macrophage markers reveals massive infiltration of TAMs in hypoxic/necrotic regions of the tumour. This massive recruitment of TAMs is usually facilitated by chemokines like CCL2, CCL5, VEGF, CSF-1, semaphorin 3A (SEMA3A), endothelin, eotaxin and oncostatin M. Once macrophages arrive in these tumour compartments, their migration is halted via hypoxiadependent mechanisms. Pro-tumoural functions of macrophages are then facilitated by a hypoxia-dependent transcription factor HIFla, which induces expression of a large set of genes associated with angiogenesis such as VEGF (Henze and Mazzone, 2016). This pro-tumoural function of hypoxic TAMs was validated by Casazza 
et al, wherein they showed that macrophage-specific genetic deletion of Nrp-1, a binding partner of hypoxia induced TAM attractant Semaphorin 3A (Sema3A), prevented macrophage entry into the hypoxic region and ablated pro-angiogenic and immunosuppressive functions of TAMs, thereby inhibiting tumour growth and metastasis (Casazza et al, 2013).

\section{TAMS IN MIGRATION AND INVASION}

The potential of tumour cells to invade and metastasise depends on the tumour microenvironment. Since TAMs constitute a major component of the tumour microenvironment, they play a crucial role in facilitating these processes. TAMs primarily promote tumour cell invasion and metastasis via secretion of matrix metalloproteinases, serine proteases, and cathepsins, which alter the composition of the ECM by modifying cell-cell junctions and promoting basal membrane disruption. For instance, high levels of cathepsin protease activity are induced in the majority of macrophages in the microenvironment of pancreatic islet cancers, mammary tumours, and lung metastases during malignant progression. Furthermore, TAM-secreted cathepsins B and S were critical for promoting pancreatic tumour growth, angiogenesis, and invasion in vivo, and also markedly enhanced the invasiveness of cancer cells in culture (Gocheva et al, 2010). According to a recent report, STAT3 and STAT6 were shown to synergistically promote cathepsin secretion by macrophages, thereby enhancing tumour invasion and metastasis. Genetic deletion of Stat3 and Stat6 impaired tumour development and invasion in vivo. Together, these findings demonstrate that STAT3 and STAT6 cooperate in macrophages and enhance tumour progression in a cathepsindependent manner (Yan et al, 2016).

Recently, Baghel and colleagues showed that a macrophagederived protein MIP-1 $\beta$ potentiated cancer cell invasion and metastasis via upregulation of MYO3A gene within breast cancer cells. Moreover, there was also a significant correlation between higher expression of this protein and poor survival of breast cancer patients, thereby validating the findings (Baghel et al, 2016). A novel real-time multiphoton imaging system developed by Wyckoff et al to investigate the metastatic nature of tumour cells demonstrated that invasion of breast cancer cells occurred in association with TAMs in mammary tumours, which is in agreement with the notion that TAMs support tumour invasion and metastasis (Wyckoff et al, 2007). In another clinicopathological study on breast carcinoma by Yang et al, the infiltration densities of TAMs were significantly higher in breast cancer patient specimens as compared to adjacent normal tissue (Yang et al, 2015). Moreover, in pancreatic tumours, targeting TAMs by inhibiting either the myeloid cell receptors colony-stimulating factor-1 receptor (CSF1R) or chemokine (C-C motif) receptor 2 (CCR2) decreased the number of tumour-initiating cells (TIC) and inhibited metastasis (Mitchem et al, 2013). A more recent study demonstrated that Warburg metabolism in tumour-conditioned macrophages promoted vascularisation, augmented extravasation of tumour cells from blood vessels, and metastasis in human pancreatic ductal adenocarcinoma. Furthermore, inhibition of glycolysis in TAMs with a competitive inhibitor disrupted this metastatic phenotype, reversing the observed increases in TAMsupported angiogenesis, extravasation, and epithelialmesenchymal transition (EMT) (Penny et al, 2016).

TAMs have also been reported to play crucial role in ovarian cancer growth, invasion and metastasis. Using an established mouse model for epithelial ovarian cancer, Yin et al showed that TAMs promote spheroid formation and tumour growth by secreting EGF. Activation of EGFR on tumour cells by EGF in turn upregulated VEGF/VEGFR signalling in surrounding tumour cells to support tumour cell proliferation and migration. Pharmacological blockade or antibody neutralisation of EGFR in TAMs abrogated spheroid formation and ovarian cancer progression in mouse models. These findings suggest that EGF secreted from TAMs plays a critical role in promoting early metastasis of ovarian cancer (Yin et al, 2016). Moreover, TAMs were also shown to promote invasion via toll-like receptor signalling in patients with ovarian cancer (Ke et al, 2016).

TAMS IN EPITHELIAL-MESENCHYMAL TRANSITION

Epithelial-mesenchymal transition plays a critical role in tumour progression and metastasis wherein polarised epithelial cells change their phenotype to motile mesenchymal cells. Recent studies have shown that TAMs are one of the orchestrators of this process, which involves loss of cell-cell contact and acquisition of a migratory phenotype. Epithelial-mesenchymal transition is characterised by suppression of epithelial markers such as E-cadherin, and upregulation of mesenchymal markers, including Vimentin, Slug, Snail, Fibronectin, zinc-finger E-box binding homeobox 1 (ZEB1), ZEB2, and $\alpha$-smooth muscle actin, as a result of which the cells acquire the ability to migrate and invade, leading to tumour progression and metastasis. Regulation of EMT is mediated by many growth factors and cytokines such as TGF- $\beta$, forkhead box protein M1 (FoxM1), hepatocyte growth factor, EGF, NFкB, Notch, and Wnt (Zhang et al, 2015). A growing body of evidence has shown the important contribution of TAMs in EMT. For instance, Liu et al showed that M2-polarised TAMs promoted EMT in pancreatic cancer cells, partially through the TLR4/IL-10 signalling pathway (Liu et al, 2013). According to another report, TAMs promote cancer stem cell (CSC)-like properties via TGF- $\beta 1$ induced EMT in hepatocellular carcinoma (Fan et al, 2014).

\section{TAMS IN INTRAVASATION AND EXTRAVASATION}

Intravasation is the process by which tumour cells enter a local blood vessel, and it is one of the important steps in the cascade of events leading to metastasis. Macrophages have been shown to enhance the ability of cancer cells to intravasate. Multiphoton intravital imaging techniques have shown that macrophages are located at the periphery of the tumour and their density decreases towards the centre wherein they are localised to the blood vessels and assist tumour cells to intravasate into the bloodstream (Sidani et al, 2006; Condeelis and Weissleder, 2010). Mechanistically, tumour cells secrete CSF1, which stimulates macrophages to produce EGF that in turn activates migration of the tumour cells (Wyckoff et al, 2004). Epidermal growth factor and CSF1 induce formation of invadopodia in cancer cells and podosomes in TAMs, structures that degrade extracellular matrix and facilitate intravasation (Condeelis and Pollard, 2006). In case of breast cancer patients, mammary TAMs secrete CCL18 which in turn triggers integrin clustering on cancer cells. This results in adherence of these cells to extracellular matrix and promotes intravasation (Chen et al, 2011a).

While in circulation, platelets form aggregates with tumour cells and protect them from cytotoxic immune cell recognition. Platelets escort tumour cells in the circulation to the site of extravasation, where they help tumour cells exit the circulation into secondary organs. According to a recent study, platelets promote extravasation of tumour cells and metastatic seeding through ATPdependent activation of the endothelial P2Y2 receptor, which opens the vessel barrier (Schumacher et al, 2013). Qian et al used an intact ex vivo lung imaging system and showed that tumour cells interacting with macrophages showed a higher percentage of 
extravasation, whereas depletion of macrophages using L-clodronate significantly reduced the number of tumour cells undergoing extravasation (Qian et al, 2009).

\section{TAMS IN THE SEED AND SOIL PARADIGM}

The seed and soil hypothesis, also known as the organ tropism hypothesis, proposed a concept that before metastatic colonisation, the primary tumour secretes factors that prepares a pre-metastatic niche at a distant site to become receptive for subsequent metastasis. It is characterised by accumulation of bone marrow (BM)-derived cell types, increased numbers of fibroblasts, secreted oncoproteins, and cytokines. Kaplan et al showed that BM-derived VEGFR + cells arrive at the distant pre-metastatic site well before the primary tumour cells arrive. Furthermore, depleting VEGFR1 + cells by antibody-mediated inhibition of VEGFR1 signalling or receptor mutation interfered with the formation of these pre-metastatic clusters and inhibited metastasis (Kaplan et al, 2005). Erler et al showed that the expression of copper-dependent amine oxidase called lysyl oxidase (LOX), which is secreted by hypoxic breast tumour cells and is a major target of hypoxiainducible factor (HIF) signalling, stabilises the ECM network by cross-linking collagen IV in the basement membranes at the premetastatic sites. This crosslinking facilitates myeloid cell recruitment and subsequent tumour cell colonisation. Moreover, LOX ablation prevents the formation of such sites and inhibits metastatic growth (Erler et al, 2009). A more recent report by Wang et al showed that colorectal carcinoma cells secrete VEGF-A, which stimulates TAMs to produce CXCL1 in the primary tumour. Elevation of CXCL1 in premetastatic liver tissue recruited CXCR2positive myeloid-derived suppressor cells (MDSC) to form a premetastatic niche that ultimately promoted liver metastases (Wang et al, 2017). Thus, TAMs play a critical role in preparing the premetastatic niche, recruitment and retention of circulating tumour cells at the metastatic site, and fostering their growth.

\section{INTERACTION WITH CANCER STEM CELLS}

Cancer stem cells are specific subpopulations of cells within tumours that exhibit stem cell-like properties and have the potential to initiate tumours by undergoing self-renewal and differentiation. Owing to the evidence of pivotal roles of stromal cells such as TAMs, the interaction between TAMs and CSCs has become an exciting area of research. Recent studies have tried to analyse functional roles of TAMs in regulating tumour promoting activities of CSCs through complex molecular networks comprised of cytokines, chemokines and growth factors (Jinushi et al, 2012). Several studies have reported interaction of macrophages with CSCs in many tumour models. Yi et al showed a positive correlation between infiltration of macrophages and glioma initiating cancer stem cells or GICSCs. TAMs were localised in higher densities in areas with a higher number of GICSCs with close contacts between the two cell types. Moreover, in glioma tissue, the secretion of CSC-derived chemoattractants such as CCL2, CCL5, VEGF-A was much higher, facilitating recruitment of macrophages (Yi et al, 2011). Another report suggested that CSCs in glioma tissue induced macrophage infiltration and polarisation into M2 phenotype because these macrophages secreted a large number of cytokines, such as TGF- $\beta 1$ and IL-10, and facilitated immunosuppression (Wu et al, 2010). Jinushi et al showed that TAMs interact with CSCs and promote their tumourigenic potential via production of milk fat globule-epidermal growth factor-VIII (MFG-E8) and IL-6 through coordinated activation of the STAT3 and sonic hedgehog pathways. Interestingly, CSC population is the major population promoting the production of MFG-E8 and IL-6 from macrophages, suggesting that they impart macrophages with the ability to produce tumourigenic factors such as MFG-E8 and IL-6 (Jinushi et al, 2011).

\section{TAMS IN IMMUNOSUPPRESSION}

The role TAMs play in immunosuppression to promote tumour progression has been widely investigated. TAMs are involved in immunosuppression either by directly inhibiting the CD8 + T-cell response through direct cell-cell interaction with $\mathrm{T}$ cells or by secreting immunosuppressive cytokines and proteases such as IL10 , TGF- $\beta$, Arginase-1, and prostaglandins, which inhibit T-cell activation and proliferation. For instance, macrophages express PD-L1/PD-L2 and CD80/CD86, which are the ligands of the inhibitory receptors programmed cell death protein 1 (PD-1) and cytotoxic T-lymphocyte antigen 4 (CTLA4), respectively. Activation of these receptors by their ligands results in inhibition of TCR signalling and T-cell cytotoxic function (Kuang et al, 2009; Ojalvo et al, 2009). Recently, it was shown that hypoxia also plays an important role in immunosuppression. TAMs found in hypoxic regions of the tumour upregulate PD-L1 expression via HIF- $1 \alpha$ signalling and consequently induce T-cell suppression (Doedens et al, 2010; Noman et al, 2014). Another report suggested that, as compared to MDSCs, macrophages produced higher levels of antiinflammatory factors and were more immunosuppressive, facilitating tumour immunoevasion in multiple murine models of breast cancers (Fang et al, 2017). CAFs were also shown to induce accumulation of TAMs and secretion of IL-10, TGF- $\beta$, and Arginase-1 in oral squamous cell carcinoma and promote an immunosuppressive microenvironment by suppressing T-cell proliferation (Takahashi et al, 2017). TAM-derived cytokines also play an important role in immunosuppression. For instance, TFG$\beta$ was shown to inhibit the anti-tumour activity of $\mathrm{CD} 8+\mathrm{T}$ cells by downregulating the expression of cytolytic genes (Thomas and Massague, 2005). Moreover, IL-10 which is expressed by TAMs, $\mathrm{CD}^{+}{ }^{+} \mathrm{T}$ cells, and tumour cells is an important cytokine in the tumour microenvironment and plays anti-inflammatory, immunosuppressive role that favours tumour escape from immune surveillance. TAM-derived IL-10 suppresses the expression of IL12, which is considered as a potential anti-tumour cytokine (Matsuda et al, 1994).

\section{TAMS AS THERAPEUTIC TARGETS}

Several reports have provided strong evidence that TAMs are crucial components of the tumour microenvironment and TAM infiltration is strongly associated with poor prognosis and survival rates in cancer patients. Based on these findings, targeting TAMs is emerging as an attractive strategy for therapeutic intervention (Chanmee et al, 2014; Yang and Zhang, 2017).

It is now well-established that chemoattractants in tumour microenvironment facilitate massive infiltration of macrophages in tumours. Hence, depletion or inhibition of TAM recruitment by modulating levels of these chemoattractants may serve as an effective strategy. Indeed, pharmacological inhibition, neutralising monoclonal antibodies, or genetic mutation of chemoattractants such as CCL2, VEGFR2, CSF-1R depleted TAM infiltration and reduced tumour growth. For instance, in case of CSF1R, the humanised monoclonal antibody RG7155 potently inhibited CSF1R dimerisation and also induced a striking reduction in the CSF1R + CD163 + macrophage population within tumour tissues (Ries et al, 2014). Moreover, PLX3397, a potent tyrosine kinase 
inhibitor of CSF1R decreased macrophage infiltration thereby enhancing the efficacy of immunotherapy (Mok et al, 2014).

Differentiation of pro-tumourigenic M2 to the anti-tumour M1 phenotype is rapidly emerging as a new therapeutic approach. Activation of TLR3/Toll-IL-1 receptor domain-containing adaptor molecule 1 by Poly (I:C) rapidly enhanced secretion of proinflammatory cytokines and accelerated M1 macrophage polarisation (Shime et al, 2012). Emerging evidence has indicated that abnormalities in tumour vasculature alter the tumour microenvironment and influences tumour progression and responses to cancer therapy. The re-education of TAMs within the tumour could restore normal vasculature and block the pro-tumourigenic effects of TAMs. Indeed, polarisation from an M2 to M1 phenotype suppressed mammary tumour growth and angiogenesis in vivo (Zhang et al, 2013). According to another report, histidinerich glycoprotein inhibited tumour growth and metastasis by inducing macrophage polarisation and vessel normalisation via downregulation of the placental growth factor (Rolny et al, 2011).

\section{CONCLUDING REMARKS}

The conventional binary model of macrophage polarisation is becoming antiquated and a newer model of polarisation spectrum is coming into conception, which focuses on involvement of an array of differentiated macrophages in various immunoregulatory disorders as well as cancers. Moreover, it is now well-established that apart from tumour cell-derived factors, stromal microenvironmental factors play a substantial role in tumourigenesis and TAMs constitute as key players in this phenomenon by regulating various steps of tumour initiation, progression, and metastasis. Since the abrogation of immunosuppressive macrophages in the tumour microenvironment enhances anti-tumour response, targeting TAMs is rapidly emerging as a promising therapeutic strategy for cancer patients.

\section{ACKNOWLEDGEMENTS}

This work was supported by National Cancer Institute, National Institutes of Health grant number R01CA193711 to M Raza Zaidi.

\section{CONFLICT OF INTEREST}

The authors declare no conflict of interest.

\section{REFERENCES}

Baghel KS, Tewari BN, Shrivastava R, Malik SA, Lone MU, Jain NK, Tripathi C, Kanchan RK, Dixit S, Singh K, Mitra K, Negi MP, Srivastava M, Misra S, Bhatt ML, Bhadauria S (2016) Macrophages promote matrix protrusive and invasive function of breast cancer cells via MIP-1beta dependent upregulation of MYO3A gene in breast cancer cells. Oncoimmunology 5(7): e1196299.

Balkwill F (2004) Cancer and the chemokine network. Nat Rev Cancer 4(7): 540-550.

Barros MH, Hauck F, Dreyer JH, Kempkes B, Niedobitek G (2013) Macrophage polarisation: an immunohistochemical approach for identifying M1 and M2 macrophages. PLoS One 8(11): e80908.

Campbell MJ, Tonlaar NY, Garwood ER, Huo D, Moore DH, Khramtsov AI, Au A, Baehner F, Chen Y, Malaka DO, Lin A, Adeyanju OO, Li S, Gong C, McGrath M, Olopade OI, Esserman LJ (2011) Proliferating macrophages associated with high grade, hormone receptor negative breast cancer and poor clinical outcome. Breast Cancer Res Treat 128(3): 703-711.

Casazza A, Laoui D, Wenes M, Rizzolio S, Bassani N, Mambretti M, Deschoemaeker S, Van Ginderachter JA, Tamagnone L, Mazzone M
(2013) Impeding macrophage entry into hypoxic tumor areas by Sema3A/ Nrp1 signaling blockade inhibits angiogenesis and restores antitumor immunity. Cancer Cell 24(6): 695-709.

Chanmee T, Ontong P, Konno K, Itano N (2014) Tumor-associated macrophages as major players in the tumor microenvironment. Cancers (Basel) 6(3): 1670-1690.

Chavez-Galan L, Olleros ML, Vesin D, Garcia I (2015) Much more than M1 and M2 macrophages, there are also $\mathrm{CD} 169(+)$ and $\mathrm{TCR}(+)$ macrophages. Front Immunol 6: 263.

Chen J, Yao Y, Gong C, Yu F, Su S, Chen J, Liu B, Deng H, Wang F, Lin L, Yao H, Su F, Anderson KS, Liu Q, Ewen ME, Yao X, Song E (2011a) CCL18 from tumor-associated macrophages promotes breast cancer metastasis via PITPNM3. Cancer Cell 19(4): 541-555.

Chen P, Huang Y, Bong R, Ding Y, Song N, Wang X, Song X, Luo Y (2011b) Tumor-associated macrophages promote angiogenesis and melanoma growth via adrenomedullin in a paracrine and autocrine manner. Clin Cancer Res 17(23): 7230-7239.

Chow A, Huggins M, Ahmed J, Hashimoto D, Lucas D, Kunisaki Y, Pinho S, Leboeuf M, Noizat C, van Rooijen N, Tanaka M, Zhao ZJ, Bergman A, Merad M, Frenette PS (2013) CD169(+) macrophages provide a niche promoting erythropoiesis under homeostasis and stress. Nat Med 19(4): 429-436.

Condeelis J, Pollard JW (2006) Macrophages: obligate partners for tumor cell migration, invasion, and metastasis. Cell 124(2): 263-266.

Condeelis J, Weissleder R (2010) In vivo imaging in cancer. Cold Spring Harb Perspect Biol 2(12): a003848.

Darwich L, Coma G, Pena R, Bellido R, Blanco EJ, Este JA, Borras FE, Clotet B, Ruiz L, Rosell A, Andreo F, Parkhouse RM, Bofill M (2009) Secretion of interferon-gamma by human macrophages demonstrated at the single-cell level after costimulation with interleukin (IL)-12 plus IL-18. Immunology 126(3): 386-393.

Doedens AL, Stockmann C, Rubinstein MP, Liao D, Zhang N, DeNardo DG, Coussens LM, Karin M, Goldrath AW, Johnson RS (2010) Macrophage expression of hypoxia-inducible factor-1 alpha suppresses T-cell function and promotes tumor progression. Cancer Res 70(19): 7465-7475.

Erler JT, Bennewith KL, Cox TR, Lang G, Bird D, Koong A, Le QT, Giaccia AJ (2009) Hypoxia-induced lysyl oxidase is a critical mediator of bone marrow cell recruitment to form the premetastatic niche. Cancer Cell 15(1): 35-44.

Fan QM, Jing YY, Yu GF, Kou XR, Ye F, Gao L, Li R, Zhao QD, Yang Y, Lu ZH, Wei LX (2014) Tumor-associated macrophages promote cancer stem cell-like properties via transforming growth factor-beta1-induced epithelial-mesenchymal transition in hepatocellular carcinoma. Cancer Lett 352(2): 160-168.

Fan X, Zhang H, Cheng Y, Jiang X, Zhu J, Jin T (2016) Double roles of macrophages in human neuroimmune diseases and their animal models. Mediators Inflamm 2016: 8489251.

Fang Z, Wen C, Chen X, Yin R, Zhang C, Wang X, Huang Y (2017) Myeloidderived suppressor cell and macrophage exert distinct angiogenic and immunosuppressive effects in breast cancer. Oncotarget 8(33): 54173-54186.

Gazzaniga S, Bravo AI, Guglielmotti A, van Rooijen N, Maschi F, Vecchi A, Mantovani A, Mordoh J, Wainstok R (2007) Targeting tumor-associated macrophages and inhibition of MCP-1 reduce angiogenesis and tumor growth in a human melanoma xenograft. J Invest Dermatol 127(8): 2031-2041.

Georgoudaki AM, Prokopec KE, Boura VF, Hellqvist E, Sohn S, Ostling J, Dahan R, Harris RA, Rantalainen M, Klevebring D, Sund M, Brage SE, Fuxe J, Rolny C, Li F, Ravetch JV, Karlsson MC (2016) Reprogramming tumor-associated macrophages by antibody targeting inhibits cancer progression and metastasis. Cell rep 15(9): 2000-2011.

Gocheva V, Wang HW, Gadea BB, Shree T, Hunter KE, Garfall AL, Berman T, Joyce JA (2010) IL-4 induces cathepsin protease activity in tumor-associated macrophages to promote cancer growth and invasion. Genes Dev 24(3): 241-255.

Gordon SR, Maute RL, Dulken BW, Hutter G, George BM, McCracken MN, Gupta R, Tsai JM, Sinha R, Corey D, Ring AM, Connolly AJ, Weissman IL (2017) PD-1 expression by tumour-associated macrophages inhibits phagocytosis and tumour immunity. Nature 545(7655): 495-499.

Halin S, Rudolfsson SH, Van Rooijen N, Bergh A (2009) Extratumoral macrophages promote tumor and vascular growth in an orthotopic rat prostate tumor model. Neoplasia 11(2): 177-186. 
Hashimoto O, Yoshida M, Koma Y, Yanai T, Hasegawa D, Kosaka Y, Nishimura N, Yokozaki H (2016) Collaboration of cancer-associated fibroblasts and tumour-associated macrophages for neuroblastoma development. J Pathol 240(2): 211-223.

Henze AT, Mazzone M (2016) The impact of hypoxia on tumor-associated macrophages. J Clin Invest 126(10): 3672-3679.

Huang YC, Feng ZP (2013) The good and bad of microglia/macrophages: new hope in stroke therapeutics. Acta Pharmacol Sin 34(1): 6-7.

Jaguin M, Houlbert N, Fardel O, Lecureur V (2013) Polarization profiles of human M-CSF-generated macrophages and comparison of M1-markers in classically activated macrophages from GM-CSF and M-CSF origin. Cell Immunol 281(1): 51-61.

Jaiswal S, Jamieson CH, Pang WW, Park CY, Chao MP, Majeti R, Traver D, van Rooijen N, Weissman IL (2009) CD47 is upregulated on circulating hematopoietic stem cells and leukemia cells to avoid phagocytosis. Cell 138(2): 271-285.

Jiang S, Yang Y, Fang M, Li X, Yuan X, Yuan J (2016) Co-evolution of tumorassociated macrophages and tumor neo-vessels during cervical cancer invasion. Oncol Lett 12(4): 2625-2631.

Jinushi M, Baghdadi M, Chiba S, Yoshiyama H (2012) Regulation of cancer stem cell activities by tumor-associated macrophages. Am J Cancer Res 2(5): 529-539.

Jinushi M, Chiba S, Yoshiyama H, Masutomi K, Kinoshita I, Dosaka-Akita H, Yagita H, Takaoka A, Tahara H (2011) Tumor-associated macrophages regulate tumorigenicity and anticancer drug responses of cancer stem/ initiating cells. Proc Natl Acad Sci USA 108(30): 12425-12430.

Joyce JA, Pollard JW (2009) Microenvironmental regulation of metastasis. Nat Rev Cancer 9(4): 239-252.

Kale S, Raja R, Thorat D, Soundararajan G, Patil TV, Kundu GC (2015) Osteopontin signaling upregulates cyclooxygenase-2 expression in tumorassociated macrophages leading to enhanced angiogenesis and melanoma growth via alpha9beta1 integrin. Oncogene 34(42): 5408-5410.

Kaplan RN, Riba RD, Zacharoulis S, Bramley AH, Vincent L, Costa C, MacDonald DD, Jin DK, Shido K, Kerns SA, Zhu Z, Hicklin D, Wu Y, Port JL, Altorki N, Port ER, Ruggero D, Shmelkov SV, Jensen KK, Rafii S, Lyden D (2005) VEGFR1-positive haematopoietic bone marrow progenitors initiate the pre-metastatic niche. Nature 438(7069): $820-827$.

Ke X, Zhang S, Wu M, Lou J, Zhang J, Xu T, Huang L, Huang P, Wang F, Pan S (2016) Tumor-associated macrophages promote invasion via Toll-like receptors signaling in patients with ovarian cancer. Int Immunopharmacol 40: 184-195.

Komohara Y, Hasita H, Ohnishi K, Fujiwara Y, Suzu S, Eto M, Takeya M (2011) Macrophage infiltration and its prognostic relevance in clear cell renal cell carcinoma. Cancer Sci 102(7): 1424-1431.

Komohara Y, Takeya M (2017) CAFs and TAMs: maestros of the tumour microenvironment. J Pathol 241(3): 313-315.

Kuang DM, Zhao Q, Peng C, Xu J, Zhang JP, Wu C, Zheng L (2009) Activated monocytes in peritumoral stroma of hepatocellular carcinoma foster immune privilege and disease progression through PD-L1. J Exp Med 206(6): 1327-1337.

Laoui D, Van Overmeire E, Di Conza G, Aldeni C, Keirsse J, Morias Y, Movahedi K, Houbracken I, Schouppe E, Elkrim Y, Karroum O, Jordan B, Carmeliet P, Gysemans C, De Baetselier P, Mazzone M, Van Ginderachter JA (2014) Tumor hypoxia does not drive differentiation of tumor-associated macrophages but rather fine-tunes the M2-like macrophage population. Cancer Res 74(1): 24-30.

Lin EY, Nguyen AV, Russell RG, Pollard JW (2001) Colony-stimulating factor 1 promotes progression of mammary tumors to malignancy. J Exp Med 193(6): 727-740.

Lin EY, Pollard JW (2007) Tumor-associated macrophages press the angiogenic switch in breast cancer. Cancer Res 67(11): 5064-5066.

Linde N, Lederle W, Depner S, van Rooijen N, Gutschalk CM, Mueller MM (2012) Vascular endothelial growth factor-induced skin carcinogenesis depends on recruitment and alternative activation of macrophages. J Pathol 227(1): 17-28.

Liu CY, Xu JY, Shi XY, Huang W, Ruan TY, Xie P, Ding JL (2013) M2polarized tumor-associated macrophages promoted epithelialmesenchymal transition in pancreatic cancer cells, partially through TLR4/ IL-10 signaling pathway. Lab Invest 93(7): 844-854.

Mahmoud SM, Lee AH, Paish EC, Macmillan RD, Ellis IO, Green AR (2012) Tumour-infiltrating macrophages and clinical outcome in breast cancer. J Clin Pathol 65(2): 159-163.
Matsuda M, Salazar F, Petersson M, Masucci G, Hansson J, Pisa P, Zhang QJ, Masucci MG, Kiessling R (1994) Interleukin 10 pretreatment protects target cells from tumor- and allo-specific cytotoxic $\mathrm{T}$ cells and downregulates HLA class I expression. J Exp Med 180(6): 2371-2376.

Matsumoto-Okazaki Y, Yamane J, Kajiya K (2015) Real-time imaging of interaction between macrophages and lymphatic vessels in an in vitro model to study inflammatory resolution. J Dermatol Sci 77(1): 76-79.

Medrek C, Ponten F, Jirstrom K, Leandersson K (2012) The presence of tumor associated macrophages in tumor stroma as a prognostic marker for breast cancer patients. BMC Cancer 12: 306.

Mitchem JB, Brennan DJ, Knolhoff BL, Belt BA, Zhu Y, Sanford DE, Belaygorod L, Carpenter D, Collins L, Piwnica-Worms D, Hewitt S, Udupi GM, Gallagher WM, Wegner C, West BL, Wang-Gillam A, Goedegebuure P, Linehan DC, DeNardo DG (2013) Targeting tumorinfiltrating macrophages decreases tumor-initiating cells, relieves immunosuppression, and improves chemotherapeutic responses. Cancer Res 73(3): 1128-1141.

Miyake M, Hori S, Morizawa Y, Tatsumi Y, Nakai Y, Anai S, Torimoto K, Aoki K, Tanaka N, Shimada K, Konishi N, Toritsuka M, Kishimoto T, Rosser CJ, Fujimoto K (2016) CXCL1-mediated interaction of cancer cells with tumor-associated macrophages and cancer-associated fibroblasts promotes tumor progression in human bladder cancer. Neoplasia 18(10): 636-646.

Mok S, Koya RC, Tsui C, Xu J, Robert L, Wu L, Graeber TG, West BL, Bollag G, Ribas A (2014) Inhibition of CSF-1 receptor improves the antitumor efficacy of adoptive cell transfer immunotherapy. Cancer Res 74(1): 153-161.

Morris PG, Hudis CA, Giri D, Morrow M, Falcone DJ, Zhou XK, Du B, Brogi E, Crawford CB, Kopelovich L, Subbaramaiah K, Dannenberg AJ (2011) Inflammation and increased aromatase expression occur in the breast tissue of obese women with breast cancer. Cancer Prev Res (Phila) 4(7): 1021-1029.

Murdoch C, Tazzyman S, Webster S, Lewis CE (2007) Expression of Tie-2 by human monocytes and their responses to angiopoietin-2. J Immunol 178(11): 7405-7411.

Nieman KM, Kenny HA, Penicka CV, Ladanyi A, Buell-Gutbrod R, Zillhardt MR, Romero IL, Carey MS, Mills GB, Hotamisligil GS, Yamada SD, Peter ME, Gwin K, Lengyel E (2011) Adipocytes promote ovarian cancer metastasis and provide energy for rapid tumor growth. Nat Med 17(11): 1498-1503.

Noman MZ, Desantis G, Janji B, Hasmim M, Karray S, Dessen P, Bronte V, Chouaib S (2014) PD-L1 is a novel direct target of HIF-1alpha, and its blockade under hypoxia enhanced MDSC-mediated T cell activation. J Exp Med 211(5): 781-790.

Ojalvo LS, King W, Cox D, Pollard JW (2009) High-density gene expression analysis of tumor-associated macrophages from mouse mammary tumors. Am J Pathol 174(3): 1048-1064.

Ong SM, Tan YC, Beretta O, Jiang D, Yeap WH, Tai JJ, Wong WC, Yang H, Schwarz H, Lim KH, Koh PK, Ling KL, Wong SC (2012) Macrophages in human colorectal cancer are pro-inflammatory and prime $\mathrm{T}$ cells towards an anti-tumour type-1 inflammatory response. Eur J Immunol 42(1): 89-100.

Penny HL, Sieow JL, Adriani G, Yeap WH, See Chi Ee P, San Luis B, Lee B, Lee T, Mak SY, Ho YS, Lam KP, Ong CK, Huang RY, Ginhoux F, Rotzschke O, Kamm RD, Wong SC (2016) Warburg metabolism in tumor-conditioned macrophages promotes metastasis in human pancreatic ductal adenocarcinoma. Oncoimmunology 5(8): e1191731.

Qian B, Deng Y, Im JH, Muschel RJ, Zou Y, Li J, Lang RA, Pollard JW (2009) A distinct macrophage population mediates metastatic breast cancer cell extravasation, establishment and growth. PLoS One 4(8): e6562.

Qian BZ, Li J, Zhang H, Kitamura T, Zhang J, Campion LR, Kaiser EA, Snyder LA, Pollard JW (2011) CCL2 recruits inflammatory monocytes to facilitate breast-tumour metastasis. Nature 475(7355): 222-225.

Qian BZ, Pollard JW (2010) Macrophage diversity enhances tumor progression and metastasis. Cell 141(1): 39-51.

Quail DF, Joyce JA (2013) Microenvironmental regulation of tumor progression and metastasis. Nat Med 19(11): 1423-1437.

Raes G, Brys L, Dahal BK, Brandt J, Grooten J, Brombacher F, Vanham G, Noel W, Bogaert P, Boonefaes T, Kindt A, Van den Bergh R, Leenen PJ, De Baetselier P, Ghassabeh GH (2005) Macrophage galactose-type C-type lectins as novel markers for alternatively activated macrophages elicited by parasitic infections and allergic airway inflammation. J Leukoc Biol 77(3): 321-327. 
Raes G, De Baetselier P, Noel W, Beschin A, Brombacher F, Hassanzadeh Gh G (2002) Differential expression of FIZZ1 and Ym1 in alternatively versus classically activated macrophages. J Leukoc Biol 71(4): 597-602.

Ries CH, Cannarile MA, Hoves S, Benz J, Wartha K, Runza V, Rey-Giraud F, Pradel LP, Feuerhake F, Klaman I, Jones T, Jucknischke U, Scheiblich S, Kaluza K, Gorr IH, Walz A, Abiraj K, Cassier PA, Sica A, Gomez-Roca C, de Visser KE, Italiano A, Le Tourneau C, Delord JP, Levitsky H, Blay JY, Ruttinger D (2014) Targeting tumor-associated macrophages with antiCSF-1R antibody reveals a strategy for cancer therapy. Cancer Cell 25(6): 846-859.

Robinson CM, O'Dee D, Hamilton T, Nau GJ (2010) Cytokines involved in interferon-gamma production by human macrophages. Journal of Innate immun 2(1): 56-65.

Rolny C, Mazzone M, Tugues S, Laoui D, Johansson I, Coulon C, Squadrito ML, Segura I, Li X, Knevels E, Costa S, Vinckier S, Dresselaer T, Akerud P, De Mol M, Salomaki H, Phillipson M, Wyns S, Larsson E, Buysschaert I, Botling J, Himmelreich U, Van Ginderachter JA, De Palma M, Dewerchin M, Claesson-Welsh L, Carmeliet P (2011) HRG inhibits tumor growth and metastasis by inducing macrophage polarization and vessel normalization through downregulation of PIGF. Cancer Cell 19(1): 31-44.

Sakai Y, Honda M, Fujinaga H, Tatsumi I, Mizukoshi E, Nakamoto Y, Kaneko S (2008) Common transcriptional signature of tumor-infiltrating mononuclear inflammatory cells and peripheral blood mononuclear cells in hepatocellular carcinoma patients. Cancer Res 68(24): 10267-10279.

Schumacher D, Strilic B, Sivaraj KK, Wettschureck N, Offermanns S (2013) Platelet-derived nucleotides promote tumor-cell transendothelial migration and metastasis via P2Y2 receptor. Cancer Cell 24(1): 130-137.

Shime H, Matsumoto M, Oshiumi H, Tanaka S, Nakane A, Iwakura Y, Tahara H, Inoue N, Seya T (2012) Toll-like receptor 3 signaling converts tumor-supporting myeloid cells to tumoricidal effectors. Proc Natl Acad Sci USA 109(6): 2066-2071.

Shree T, Olson OC, Elie BT, Kester JC, Garfall AL, Simpson K, Bell-McGuinn KM, Zabor EC, Brogi E, Joyce JA (2011) Macrophages and cathepsin proteases blunt chemotherapeutic response in breast cancer. Genes Dev 25(23): 2465-2479.

Sica A, Saccani A, Mantovani A (2002) Tumor-associated macrophages: a molecular perspective. Int Immunopharmacol 2(8): 1045-1054.

Sidani M, Wyckoff J, Xue C, Segall JE, Condeelis J (2006) Probing the microenvironment of mammary tumors using multiphoton microscopy. J Mammary Gland Biol Neoplasia 11(2): 151-163.

Strachan DC, Ruffell B, Oei Y, Bissell MJ, Coussens LM, Pryer N, Daniel D (2013) CSF1R inhibition delays cervical and mammary tumor growth in murine models by attenuating the turnover of tumor-associated macrophages and enhancing infiltration by $\mathrm{CD} 8+\mathrm{T}$ cells. Oncoimmunology 2(12): e26968.

Takahashi H, Sakakura K, Kudo T, Toyoda M, Kaira K, Oyama T, Chikamatsu K (2017) Cancer-associated fibroblasts promote an immunosuppressive microenvironment through the induction and accumulation of protumoral macrophages. Oncotarget 8(5): 8633-8647.

Thomas DA, Massague J (2005) TGF-beta directly targets cytotoxic T cell functions during tumor evasion of immune surveillance. Cancer Cell 8(5): 369-380.

Vicetti Miguel RD, Cherpes TL, Watson LJ, McKenna KC (2010) CTL induction of tumoricidal nitric oxide production by intratumoral macrophages is critical for tumor elimination. J Immunol 185(11): 6706-6718.

Wang D, Sun H, Wei J, Cen B, DuBois RN (2017) CXCL1 is critical for premetastatic niche formation and metastasis in colorectal cancer. Cancer Res 77(13): 3655-3665.
Wang T, Xiao M, Ge Y, Krepler C, Belser E, Lopez-Coral A, Xu X, Zhang G, Azuma R, Liu Q, Liu R, Li L, Amaravadi RK, Xu W, Karakousis G, Gangadhar TC, Schuchter LM, Lieu M, Khare S, Halloran MB, Herlyn M, Kaufman RE (2015) BRAF inhibition stimulates melanoma-associated macrophages to drive tumor growth. Clin Cancer Res 21(7): 1652-1664.

Werchau S, Toberer F, Enk A, Dammann R, Helmbold P (2012) Merkel cell carcinoma induces lymphatic microvessel formation. J Am Acad Dermatol 67(2): 215-225.

Wu A, Wei J, Kong LY, Wang Y, Priebe W, Qiao W, Sawaya R, Heimberger AB (2010) Glioma cancer stem cells induce immunosuppressive macrophages/microglia. Neuro Oncol 12(11): $1113-1125$.

Wyckoff J, Wang W, Lin EY, Wang Y, Pixley F, Stanley ER, Graf T, Pollard JW, Segall J, Condeelis J (2004) A paracrine loop between tumor cells and macrophages is required for tumor cell migration in mammary tumors. Cancer Res 64(19): 7022-7029.

Wyckoff JB, Wang Y, Lin EY, Li JF, Goswami S, Stanley ER, Segall JE, Pollard JW, Condeelis J (2007) Direct visualization of macrophage-assisted tumor cell intravasation in mammary tumors. Cancer Res 67(6): 2649-2656.

Wynn TA, Chawla A, Pollard JW (2013) Macrophage biology in development, homeostasis and disease. Nature 496(7446): 445-455.

Xue J, Schmidt SV, Sander J, Draffehn A, Krebs W, Quester I, De Nardo D, Gohel TD, Emde M, Schmidleithner L, Ganesan H, Nino-Castro AMallmann MR, Labzin L, Theis H, Kraut M, Beyer M, Latz E, Freeman TC, Ulas T, Schultze JL (2014) Transcriptome-based network analysis reveals a spectrum model of human macrophage activation. Immunity 40(2): 274-288.

Yan D, Wang HW, Bowman RL, Joyce JA (2016) STAT3 and STAT6 signaling pathways synergize to promote cathepsin secretion from macrophages via IRE1alpha activation. Cell Rep 16(11): 2914-2927.

Yang J, Li X, Liu X, Liu Y (2015) The role of tumor-associated macrophages in breast carcinoma invasion and metastasis. Int J Clin Exp Pathol 8(6): 6656-6664.

Yang L, Zhang Y (2017) Tumor-associated macrophages: from basic research to clinical application. J Hematol Oncol 10(1): 58.

Yi L, Xiao H, Xu M, Ye X, Hu J, Li F, Li M, Luo C, Yu S, Bian X, Feng H (2011) Glioma-initiating cells: a predominant role in microglia/ macrophages tropism to glioma. J Neuroimmunol 232(1-2): 75-82.

Yin M, Li X, Tan S, Zhou HJ, Ji W, Bellone S, Xu X, Zhang H, Santin AD, Lou G, Min W (2016) Tumor-associated macrophages drive spheroid formation during early transcoelomic metastasis of ovarian cancer. J Clin Invest 126(11): 4157-4173.

Zaidi MR, Davis S, Noonan FP, Graff-Cherry C, Hawley TS, Walker RL, Feigenbaum L, Fuchs E, Lyakh L, Young HA, Hornyak TJ, Arnheiter H, Trinchieri G, Meltzer PS, De Fabo EC, Merlino G (2011) Interferongamma links ultraviolet radiation to melanomagenesis in mice. Nature 469(7331): 548-553.

Zhang J, Yao H, Song G, Liao X, Xian Y, Li W (2015) Regulation of epithelialmesenchymal transition by tumor-associated macrophages in cancer. $\mathrm{Am} \mathrm{J}$ Transl Res 7(10): 1699-1711.

Zhang X, Tian W, Cai X, Wang X, Dang W, Tang H, Cao H, Wang L, Chen T (2013) Hydrazinocurcumin Encapsuled nanoparticles 're-educate' tumorassociated macrophages and exhibit anti-tumor effects on breast cancer following STAT3 suppression. PLoS One 8(6): e65896.

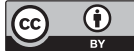

This work is licensed under the Creative Commons Attribution 4.0 International License. To view a copy of this license, visit http://creativecommons.org/licenses/by/4.0/

C) The Author(s) named above 2017 